\title{
MASCEM: Optimizing the Performance of a Multi-Agent System
}

\author{
Gabriel Santos ${ }^{1}$, Tiago Pinto ${ }^{1, *}$, Isabel Praça ${ }^{1}$, Zita Vale $^{1}$ \\ ${ }^{1} G E C A D$ - Research Group on Intelligent Engineering and Computing for Advanced Innovation and \\ Development - School of Engineering - Polytechnic of Porto (ISEP/IPP) \\ R. Dr. António Bernardino de Almeida, 431, 4200-072 Porto, Portugal

\section{ABSTRACT}

The electricity market sector has suffered massive changes in the last few decades. The worldwide electricity market restructuring has been conducted to potentiate the increase in competitiveness and thus decrease electricity prices. However, the complexity in this sector has grown significantly as well, with the emergence of several new types of players, interacting in a constantly changing environment. Several electricity market simulators have been introduced in recent years with the purpose of supporting operators, regulators, and the involved players in understanding and dealing with this complex environment. This paper presents a new, enhanced version of MASCEM (Multi-Agent System for Competitive Electricity Markets), an electricity market simulator with over ten years of existence, which had to be restructured in order to be able to face the highly demanding requirements that the decision support in this field requires. This restructuring optimizes the performance of MASCEM, both in results and execution time.

KEYWORDS: Decision Support Systems; Electricity Markets; Modelling; Multi-Agent Systems; Simulation

\section{Introduction}

All over the world the electricity markets' restructuring placed several challenges to governments and companies that are involved in generation, transmission and distribution of electrical energy. Potential benefits, however, depend on the efficient operation of the market. Definition of the market structure implies a set of

\footnotetext{
* Corresponding author: Tiago Pinto is with GECAD - Research Group on Intelligent Engineering and Computing for Advanced Innovation and Development, R. Dr. António Bernardino de Almeida, 431, 4200-072 Porto, Portugal; Tel.: +351 22 8340500; Fax: +351 22 8321159, Website: http://www.gecad.isep.ipp.pt / Email: tmcfp@isep.ipp.pt
} 
complex rules and regulations that should not encourage strategic behaviours that might reduce market performance and lead to market power inefficiency [1].

Real-world restructured electricity markets are sequential open-ended games with multiple participants trading for electric power. Market players and regulators are very interested in foreseeing market behaviour: regulators to test rules before they are implemented and to detect market inefficiencies; market players to understand market behaviour and operate in order to maximize their profits [2].

The restructuring turned electricity markets into an attractive domain for developers of software tools [39]. Simulation and Artificial Intelligence techniques become essential under this context. Multi-agent based simulation is particularly well fitted to analyse dynamic and adaptive systems with complex interactions among its constituents [3]. This is supported by the several multi-agent modelling tools that can be fruitfully applied to the study of restructured wholesale power markets. Some relevant tools in this domain are AMES (Agentbased Modelling of Electricity Systems) [5], EMCAS (Electricity Market Complex Adaptive System) [6] and MASCEM (Multi-Agent Simulator of Competitive Electricity Markets) [3, 4].

MASCEM is a modelling tool that has been firstly introduced to the scientific community in 2003 [3] to allow the study of restructured electricity markets. As market players are complex entities, with particular characteristics and objectives, making their decisions and interacting with other players, a multi-agent architecture is used and proved to be adequate. Experimental results show that a coherent market behaviour emerges from the simulated environment $[4,10]$.

Since MASCEM was firstly introduced, many changes have occurred in the electricity markets field. The increase in competitiveness brought by the restructuring process, together with the increasing need to accommodate large scale distributed generation from renewable sources (such as wind and solar generation), the transformation towards the unification of regional markets [11], the need for an active participation from the consumers side, and the introduction of new paradigms (e.g. smart grids [12]) and players (e.g. Virtual Power Players [10]), bring an exponential need for simulation and decision support capabilities that cannot be easily fulfilled with old and outdated models and architectures.

Realistic electricity market simulators, capable of providing scenarios based on real data, are an enormous asset for the study of electricity markets. Market operators and regulators are able to experiment and test new market rules and mechanisms, which could not be tested directly in reality due to the impact that such 
experiments could have for the global population, and obtain valuable insights regarding the consequences of such changes, both in what affects the market itself and also in what way it influences the market players.

Electricity market simulators also provide the means for supporting electricity market participant players in their decisions, so that they can be able to take the best advantage from the market environment, by testing different strategic behaviours, and analysing their results. Real market players are also able to thoroughly studying competitor players' actions, coming to understand how they behave, act and react in different circumstances and contexts, meaning an invaluable tool for adapting their own behaviours to the expected actions from competitors. This ultimately leads to the achievement of higher profits from sellers, and the decrease of purchase costs from buyers.

Besides the advantages for market players, regulators, and operators, also students, researchers, and ultimately, the end energy consumers can benefit from electricity market simulators as well, with the potential price decrease brought by the increase in competitiveness and also from the improved energy use efficiency that may result from simulation studies. "Since MASCEM is being developed by GECAD, which is a research group from the School of Engineering of the Polytechnic of Porto, students play an important role in the development of MASCEM. They use MASCEM to explore the current market models and mechanisms in Europe, and learn how they work and what are the impacts of the participation of different types of players. Students also take advantage of the simulator's different market types, to perform comparison studies and reach conclusions on the different outcomes achieved by using different market models, or even by performing slight changes in market rules (such as offer types and complex conditions). The use of real data in MASCEM simulations is also essential for practitioners, as it allows exploring the impacts of considering different levels if penetration of players with different characteristics and behaviours (e.g. generators based on different sources). The real data based simulations also allow experimenting the potential effects of changing the market models of a certain market operator (e.g. what would happen if MIBEL started using the market models from EPEX of Nord Pool). This can be studied using the exact real players that participate in MIBEL, under altered market conditions. Finally, the possibility for co-simulation with smart grids, provides students with the chance to further explore the possible pathways of global energy markets, by studying possible alternative scenarios of integration of markets at a local level, besides the spread of market mechanisms, towards the unification of electricity markets." 
Given that MASCEM is a simulation tool with potential to be used by entities of very different natures, and consequently with very different scopes of study, it is important that the simulator is able to suit the simulation capabilities to each context of use. For this reason, an adequate balance between what is important to be executed at each time, and the execution time degradation that the execution of each different module brings, is essential. The decision support to the participation in real time or near real time markets, such as balancing markets [13] or ancillary services [14], which require the submission of actions to the market in a matter of minutes (usually every five minutes) [14], make the fast execution times of MASCEM (around one minute per simulated day without decision support features for players' actions, as shown in section IV) seem less fast when several alternative scenarios need to be timely experimented. Considering the inclusion of decision support capabilities, the achievement of results in useful time becomes even more challenging, and requires a careful balancing between the execution time that is available, and the quality of results that is provided. Another critical context of use is the simulation of smart grids, considering the planning, management and scheduling of distributed energy resources, such as wind and solar based generation, different types of consumers, and the inclusion of plug-in electric vehicles (EVs) [15]. This type of planning is usually done with some time in advance, but it needs to be adapted in near real time due to the difficulty in forecasting highly volatile resources from very intermittent natures, such as the wind speed, or the charge/discharge of EVs. In these cases, the available minutes need to be used for the forecasting, scheduling and optimization of the resources, while the market simulation is secondary and needs to provide quick results, even if only indicative of the expected market prices, in order to feed the optimization processes. Even when considering a day-ahead planning, and due to the mentioned difficulty in achieving good quality forecasts for many resources in advance (the larger the advance of the planning, i.e. the longer time available to execute simulations, the worst quality of forecasts will be achieved [16]), the execution time problem still persists, since the uncertainty of the performed forecasts requires the execution of many alternative scenarios, with different players' participation, with different players' behaviours, with different characteristics and penetration of players. Hence, apparently fast execution times do not represent an optimal response for all cases, as there are many contexts of use that require extremely fast responses that come within the required timings, even if that represents a certain degree of degradation in the quality of simulated results. 
This paper presents a novel version of the MASCEM simulator, characterized by a drastic change in the architectural approach, which aims at easily supporting the integration of new and complementary models, from diverse natures. The facilitation in accommodating different tools and mechanisms is provided by important structural implementation decisions, making MASCEM able to deal with the constantly changing and highly demanding environment of electricity markets. MASCEM's restructuring brings the use of ontologies to support players' communications $[17,18]$, providing the means for an easier cooperation with external agent societies that come to complement MASCEM's simulation capabilities. Important examples are ALBidS (Adaptive Learning strategic Bidding System) [19], which provides decision support capabilities to market negotiating players; and MASGriP (Multi-Agent Smart Grid Platform) [20], a multi-agent platform that supports the simulation of micro and smart grids.

Several implementation decisions, such as the use of parallel computing, the careful choice of programming languages for each different algorithm depending on its purpose and requirements, the intelligent distribution of agents by the machines available in the network, the use of heuristic methods when necessary, and the integration of a mechanism to manage the balance between the efficiency and effectiveness (2E) of the system, come to significantly decrease simulations execution time when such is necessary.

Finally, the use of real electricity markets data, gathered from the websites of several market operators, in real time, provides the new version of MASCEM the chance to simulate realistic scenarios that transpose the reality, just as it occurs.

The contribution of this paper is a multi-agent based model that optimizes both the effectiveness and computational efficiency of electricity markets' simulation. The efficiency of the system is achieved by managing the balance between the execution time and the quality of results depending on the necessities of each simulation; and the effectiveness of the simulation is accomplished by the system's architecture, which facilitates the integration of several models and distinct systems, enabling more realistic, flexible and comprehensive simulations. This contribution is, therefore, not a single model, directed to a specific problem, but a combination of several modules and systems, which together form a collaborative network that enables an enhanced multi-agent based simulation performance.

After this introductory section, Section II presents an overview of the related work concerning multi-agent simulation of electricity markets. Section III outlines the main features of the novel version of the MASCEM 
simulator, including the multi-agent model, the architectural and structural implementation decisions, the introduction of the use of ontologies in agents' communications, and the integration of the automatic dataextraction tool and of the 2E balance management mechanism. Section IV presents a case study, in which the novel MASCEM simulator capabilities are tested and demonstrated under two perspectives: (i) the capability in simulating real environments, even with a reduced number of participating agents; and (ii) the simulations' execution time. Finally, Section V presents the most relevant conclusions and future work.

\section{Related Work}

Multi-agent technology is being increasingly used to represent, model and simulate complex and dynamic environments [3-10, 21]. Each agent can represent a physical or virtual entity acting according their perception of the environment, behaviour profile and goals. The autonomy, the perception of the other players' actions, and the use of artificial intelligence algorithms are the most important characteristics of each agent. These characteristics turn the multi-agent simulation platforms much more complex than standard distributed simulation tools [22]. The possibility of representing different entities as independent software agents with their own particular behaviour and objectives; and the opportunity for easily enlarging the represented models, are some of the main reasons why multi-agent technology is widely chosen as the best option for developing complex simulation tools for constantly evolving environments such as the electricity markets.

The multi-agent systems can be used in a more general view in cyber-physical systems due to their capacity to manage and control real equipment and devices, taking decisions according the context and also the human interaction. Each agent can be developed in a different programming language following their own syntax according the hardware requirements. An upper level ontology should be defined and used as a middleware to provide a common communication channel for all agents [23]. In some cases, a facilitator agent is necessary to control the information flow and most important, to assure the confidentiality and authentication of the communication between agents. The upper ontology allows the communication between agents inside a multiagent platform but also the communication between different multi-agent platforms. A good example this interoperability is the interaction between the agents of MASCEM, MASGriP and ALBidS. It is important to refer that MASGriP interacts with real hardware (loads, distributed generators, storage systems, real-time digital 
simulator device and control devices based in android and windows) using different communication protocols (TCP-IP, Modbus, RS-485, ZigBee, etc.) [24].

\subsection{Multi-agent simulators}

SEPIA (Simulator for Electric Power Industry Agents) [25] is based on a Plug and Play architecture, offering the possibility of using several processing units to perform simulations, distributing simulations

through several machines inside a network. The interactions and behaviours of all players of a simulation can be followed and studied in SEPIA.

EMCAS (Electricity Market Complex Adaptive System) [6] models the market players using software agents. Players' bidding strategies are based on adaptive machine learning algorithms. EMCAS allows performing simulations for time-horizons that reach several decades.

Power Web [26] is a Web based platform, which enables users to interact from distinct geographical points, offering the possibility of participating in an open market, in which users can compete with players controlled by other users or by virtual entities.

SREMS (Short - Medium run Electricity Market Simulator) [27] is especially dedicated to the Italian electricity market. SREMS uses a game theory based scenario analysis algorithm with the objective of calculating negotiating players' optimal hourly bids.

AMES (Agent-based Modelling of Electricity Systems) [5] is directed to the study of the U.S. Federal Energy Regulatory Commission (FERC) market design. Generators' bidding decisions in the electricity market are supported by stochastic reinforcement learning algorithms.

GAPEX (Genoa Artificial Power Exchange) [7] is able to reproduce the market clearing procedures of the most important European power-exchange markets. This agent-based framework is highly directed to the study of the power exchanges between electricity market negotiating entities.

MASCEM (Multi-Agent Simulator for Competitive Electricity Markets) [3, 4] has been firstly introduced to the scientific community in 2003 [3]. The first version of MASCEM was implemented on top of OAA (Open Agent Architecture) and included a rather reduced set of different players and market models. Since then MASCEM has been accommodating several functionalities in order to cope with the constant evolution of the electricity markets environment. MASCEM is able to recreate a high diversity of market clearing models, based 
on the mechanisms used in different countries all around the world [13, 28, 29] and even the representation of possible evolution of electricity markets [30]. The introduction of Virtual Power Players (VPPs) has been presented in [4], and the simulation of a smart grid environment has been approached in [10]. The contemplation of decision support to market players in MASCEM's scope has been firstly approached in [19], and later in [31] with the introduction of ALBidS.

The continuous development of MASCEM has been, and still is, important for the study and decision support in electricity markets. However, with the constant updates MASCEM has accommodated, the multiagent environment has become overly complex, and has uncovered much fragility in the old dated architecture and on the agents' communication platform (OAA), which have started to become barely capable of supporting the evolution of the system. For this reason, a complete restructuring of the system has become essential, including the definition of the multi-agent model, the system's implementation architecture, and the use of proper mechanisms to deal with the highly demanding execution time requirements. This paper deals with these gaps, by presenting a novel, restructured version of MASCEM.

\subsection{MIBEL - Iberian market overview}

The typical EM environment consists of a day-ahead pool (symmetric or asymmetric) where energy for the following day is negotiated. Typically, a floor for bilateral contracts is also considered [32]. Moreover, intraday markets are required to provide the means to renegotiate the previously traded power in order to meet the required adjustments towards the feasibility of the daily program and of the last scheduling [13].

The MIBEL day-ahead market consists of 24 hourly periods per day. The Iberian system is treated as a single system defining the same market price for both Portugal and Spain. However, when there are congestions on the interconnection between both areas, a split mechanism is used, enabling the best possible use of the available interconnections capacity, which may result in a distinct market price per area [33].

A buying or selling bid can be carried out based on 25 offers per period. Regular bids feature for each offer a price and amount of power. If it is a selling bid, the price shall increase in each offer, while if it is a buying bid the price must decrease. Intentions of selling energy can still be accompanied by certain restrictions denominated complex conditions. 
Regarding the intraday (or balancing) market, although being very similar to the daily market, it contains 6 market sessions, where players can renegotiate previously negotiated periods in the spot market, in order to fit their needs. The first session of the market sets the price adjustments for the last 3 hours of the trading day and for the 24 hours of the following day; in the second session the price for the 24 hours of the following day may be adjusted; the third session sets the adjustments for 20 hours: between the 5 th and the 24 th hour of the following day; the fourth session adjusts the price for the 17 hours between the 8 th and the 24th hour of the following day; the fifth session adjusts the price for the 13 hours between the 12 th and 24 th hour of the following trading day; and finally, the sixth session sets the price adjustments for the 9 hours between the 16th and 24th hour of the following trading day [34]. In this market type buyers are allowed to sell and sellers are allowed to buy.

MIBEL delivers monthly reports with information about the market results, characterization, energy placed, its evolution, etc. Figure 1 shows data about the energy negotiated in the daily market for October 2015 , as seen in the MIBEL website [35].

Data on the energy placed in the day-ahead market, October 2015 [35]

Fig. 1

As it can be easily seen the main energy sources placed in the market in October 2015, for both Portugal and Spain, are from Renewables \& Cogen sources.

\section{MASCEM Restructuring}

Since its first appearance in 2003, MASCEM has come to accommodate the modelling of a huge number of different players and market types. MASCEM's restructuring aims at optimizing the performance of this multi-agent simulator, providing the means to cope with an evolving complex dynamic reality in order to provide players with adequate tools to adapt themselves to the new reality, gaining experience to act in the frame of a changing economic, financial, and regulatory environment. With this renewed and enhanced multiagent simulation tool the model may be easily enlarged and future evolution of markets may be accomplished. The integration of different models and the interconnection with other systems, with their own social environment are some of the most important advantages of multi-agent based platforms, which are potentiated by this renovation. 


\subsection{Architecture}

The definition of MASCEM's global structure has been based on a careful analysis on how the system should behave, both in an internal, independent perspective, and also in what concerns its connection and efficient communication with external multi-agent systems. For that it has been necessary to consider in a high account the design of its structure in terms of programming issues, while at the same time guaranteeing the higher possible performance in what concerns the agent's interconnectivity and communications.

When re-designing MASCEM, high importance had to be given to defining its architecture. The architecture depends on the characteristics of the system, and will influence all its conception. Choosing the MVC (Model-View-Controller) architecture ensures the independence between the data (model), the user interface (view), and the business layer (controller).

A clear separation between the user interface, the data, and the business layer, makes it possible to develop, change, and update each different component independently, without affecting the others. This facilitates the integration of different models and tools, which can be easily developed as independent platforms, and then used by MASCEM without the need to make changes to the code. A reference example is the user interface, which is loaded automatically from information stored in a XML file. This allows the interface to be changed at any time, with no influence on the performance of the system. The user interface is also dynamic, in a way that it is only used when required. In situations when less experienced users use MASCEM, or for demonstration purposes, the user interface is activated. Otherwise, when the main requirement is the optimization of the system's performance from an execution time perspective, the user interface is omitted, reducing the execution time, and the inputs can be loaded from a XML file as well, or alternatively, from an excel template file. This dependency injection culminates in the automatic loading of configurations, which can be altered off-line, facilitating the control of the system.

Restructuring a highly complex system such as MASCEM in a way that it becomes a solid and adequately structured simulation platform, requires the use of good software engineering practices. Several design patterns have been used [36, 37], namely some of the original Gang of Four (GoF) design patterns, listed in the book $[36]$.

Some examples of the used structural patterns are: Adapter, which allows classes with disparate interfaces to work together by creating a common object by which they may communicate and interact; and the Facade, 
which creates a single interface for a set of interfaces within a system. This allows layering systems and subsystems with many dependencies between each other.

Some behavioural design patterns have also been fundamental for the restructuring of MASCEM, by allowing taking advantage of classes' polymorphism, namely: Strategy, which defines a set of encapsulated algorithms that can be swapped to carry out a specific behaviour. This is crucial for the implementation of several alternative bidding strategies, such as the ones used by ALBidS. This way the behaviour of a class (in this case, the desired strategic approach) can be defined at runtime; Observer, which lets one or more objects be notified of state changes in other objects within the system. This highly facilitates the sensing capabilities of the agents within the system, enabling agents to perform their actions in the most suitable timings, by being aware of certain changes that occur in other parts of the multi-agent society.

From the creational group of design patterns, the Factory has been used; this pattern provides an interface that delegates creation calls to other concrete classes in order to deliver specific objects. The creation of MASCEM negotiating players, and of ALBidS' strategies is performed using a Factory implementation.

\subsection{Multi-Agent Model}

One of the most important outcomes of MASCEM's restructuring process is the compliance with the FIPA (Foundation for Intelligent Physical Agents) standards [38], allowing the integration with external platforms.

FIPA is devoted to develop and promote open specifications that support interoperability among agents and agent-based applications [38]. Multi-agent systems using FIPA's standards should be able to interoperate, however, it does not mean that the agents are able to share useful information due to the employment of different ontologies.

FIPA proposes the Agent Communication Language (ACL) as a standard for communications between agents. The content of the message includes the content language and the ontology. The former specifies the syntax, while the latter provides the semantics of the message [39]. This way the correct interpretation of the meaning of the message is assured, removing the ambiguity about the content. The FIPA-SL content language is the only one that reached a stable standard. Ontologies are used by agents for exchanging information, ask questions, and request the execution of actions related to their specific domain. 
Coping with FIPA standards meant implementing MASCEM's agent society in JADE (Java Agent DEvelopment Framework) [40], a development framework that simplifies the implementation of multi-agent systems, and supports the majority and most important FIPA specifications. This way MASCEM is able to interact with other multi-agent systems using a common language. However, so that messages and all the concerned concepts can be understood by different systems it is also necessary that they share a common vocabulary and semantics. To this end, ontologies are used, enabling the standardization of communications and interpretation of concepts between independent systems.

In recent years, MASCEM's multi-agent architecture has been largely expanded in order to accommodate the simulation of a diversity of player types [4, 10]. Virtual Power Players (VPPs) represent alliances of small producers, mainly based on distributed generation and renewable sources, and small consumers which are not able to compete in the market on equal footing with the big companies. VPPs manage their aggregates information, and negotiate in the market on their behalf. They also need to negotiate with players in order to establish contract conditions, and determine the revenues or costs of each aggregated player, depending on the outcomes of VPPs' participation in electricity markets. VPPs' operation has been modelled as an independent society within MASCEM's environment. Additionally, a huge number of different small players' types started to be required; some examples are electric vehicles, different types of production units, and different types of consumers (e.g. domestic, small commerce, rural consumers).

The large number of players acting in the frame of MASCEM, together with the inclusion of decision support capabilities with the integration of ALBidS [19, 31], yet another multi-agent system; signified a big increase in the complexity MASCEM's multi-agent model, and with it, a huge degradation of the execution time (over twenty times slower when considering a single player using ALBidS as decision support). Although each different agent community (MASCEM's market environment, VPPs' operation, and ALBidS) has been implemented in a way it should be as independent from the other as possible, including the implementation of specific facilitators to manage each of the different systems' communications to make them as independent and parallel as possible; the limitations of OAA were highly uncovered.

The transition to JADE facilitated the interaction with different, external, multi-agent systems, and eliminated the previous restraints concerning the use of multiple parallel facilitators to manage each independent agent society. This potentiated the definitive separation of the different systems, making each more 
efficient in its purposes, interacting with the other only in the required measure, therefore diminishing the overflow of communications within the system that had little or none importance for specific groups of the agent community (e.g. the communications between agents of ALBidS do not bring any valuable information for the market operator, only for the market players negotiating players that are using the decision support capabilities; and the communications between VPPs and their aggregates are not important for players outside the coalition).

The separation of the different agent communities, and the development of MASGriP [20], which came to support the simulation of the smaller players that act at a smart grids' level, potentiated a huge reduction and simplification of MASCEM's multi-agent model. MASCEM allows the participation in the wholesale market of aggregators representing those smart grids, as can be seen in [41] and [42]. MASCEM's market models can also be applied to transactions at the local (smart grid) level, being used to support the negotiations between small players, as detailed in [43].

The new version of MASCEM includes only five different types of agents, besides the ones provided by JADE to control the user interfaces, and to manage communications, much like the previous version facilitators. The five agent types are:

- Main Agent - enables the user's interaction with the system. It is responsible for starting the market entities from the input file or user's interface; for converting the input data into the respective RDF knowledge bases and for sending them to the respective players and operators; for distributing the various agents by the machines available for the simulation, considering the machines' features and the agents' processing needs; and for properly killing the agents when the user decides to terminate the application;

- MIB Agent - (using the SNMP protocol) it is responsible for reading the management information base of each machine, creating a report and sending it to the Main Agent so that it can decide which agents will move to each machine, as explained in section 3.4;

- Market Operator - regulates pool negotiations by validating and analysing the players' bids depending on the type of negotiation, and determines the market price, the accepted and refused bids, and the economical dispatch that will be sent to the system operator; 
- System Operator (ISO) - examines the technical feasibility from the power system point of view and solves congestion problems that may arise. It is responsible for the system's security as well as to assure that all conditions are met within the system;

- $\quad$ Player - represents buyer, seller or aggregator agents. On one hand, it may be a consumer or distribution company which participates in the EM in order to buy certain amounts of power. On the other hand, it may simulate electricity producers or other entities able to sell energy in the market, or even aggregations of several entities.

These core agents, much like the first version of MASCEM, in 2003 [3], allow a simple, yet effective electricity market simulation. More complex and advanced simulation studies are achieved through the collaboration between the different multi-agent systems, as presented in Figure 2.

Collaboration between independent multi-agent systems

As can be seen by Figure 2, the core simulation environment provided by MASCEM can be extended by the integration of VPPs, or even other small buyers and sellers that are part of a VPP coalition but wish to participate in market negotiations on their own. Other players, which act in a smart grid environment in the scope of MASGriP, or that are part of other multi-agent simulators of electricity markets, are also able to participate in MASCEM's electricity market negotiations. All these players can use the decision support capabilities provided by ALBidS.

Regarding the market models, MASCEM allows the simulation of several market types: day-ahead pool (asymmetric or symmetric, with or without complex conditions), bilateral contracts, balancing market, forward markets and ancillary services. Hybrid simulations are also permitted by selecting a combination of the market models mentioned above. Some other market types can be provided by different systems. A reference example is that of MASGriP [20]. Players in MASCEM are able to take part in smart grid related negotiations by participating in MASGriP simulations. The collaboration between the diversity of agent societies is achieved by using an upper-ontology, which defines the main concepts that must be understood by agents that participate in power systems and electricity markets' related simulations. 


\subsection{Ontologies for Multi-Agent Systems Interoperability}

Mapping all the concepts that different systems share, so that agents from different environments can properly understand and cooperate with each other, is not a trivial task [18]. In an attempt to solve this problem FIPA suggests the use of an ontology agent that provides a set of related services [44]. However, the mappings must still be done by ontologies' designers, increasing the human effort required and costs of implementation.

Alternatively, [45] proposes the use of an upper ontology representing the general concepts of the domain, ensuring a common basis for the representation of those concepts and their relations between systems while reducing the complexity of ontology mapping.

In [23, 41 a high level-ontology has been proposed, which contains the main concepts that are required by entities that participate in electricity market and power system simulations. This ontology is used by MASCEM in order to guarantee a proper collaboration with agents from other multi-agent societies, enabling knowledge exchange between them in order to take full advantage of their functionalities, and promoting the adoption of a common semantic that enables the communication between heterogeneous systems.

\subsection{Execution Time Optimization}

The diversity of algorithms and approaches that are used by MASCEM brings out the need for the development of a mechanism that is able to manage the balance between the Efficiency and Effectiveness (2E) of the system. This mechanism provides the means for the system to adapt its execution time to the purpose of the simulation, i.e., if the expected results from the simulation are as best as it is able to achieve, or, on the other hand, if the main requirement is for the system to be executed rapidly, since the purpose of the considered simulation is to analyse issues other than player's optimal performance in the electricity market. The $2 \mathrm{E}$ Management mechanism manipulates the strategies both externally and internally. From the system's perspective this mechanism contributes by deciding which tools are used at each moment for each circumstance; depending on their observed performance in terms of efficiency and effectiveness. This way this mechanism can choose to exclude certain strategies when they are not fulfilling the requirements for the case in matter. The algorithms that are chosen to be executed are also manipulated internally, so that they can adapt their individual results quality/execution time balance to the needs of each on-going simulation. 
The adaptation process is performed by means of a fuzzy process $[46,47]$. Two dynamic fuzzy variables characterize the efficiency and the effectiveness of each strategy. The characterization is what concerns the efficiency of each strategy comprises the difference between each strategy's execution time, and the reference execution time of the simulation without the use of decision support. This means that the higher the difference is, i.e. the longer a strategy takes to achieve results, when compared to the reference simulation time, a worse classification is attributed to the strategy. Regarding the characterization of the efficiency of each strategy, the quality of the forecasts is analysed, comparing the forecasted value, and the actual market price that was verified. The confusion matrix that combines the two fuzzy variables, plus the preference value of the user for a faster or better decision support, determines which strategies must be excluded from the system, for taking too long to achieve not so good results, or which must adapt their execution times, reducing them in by a certain amount. The internal adaptation of each strategy concerning the execution time is dependent on the characteristics of each strategy (e.g. a neural network can reduce the training data, to achieve faster, yet worse, results; the game theory strategy can reduce the number of considered scenarios; the optimization based strategies can use heuristic processes rather than deterministic approaches, in order to achieve faster results, even if only near-optimal).

The dynamism of the fuzzy process is achieved by adapting the fuzzy membership functions in execution time, as new events occur. This means that when each MASCEM simulation ends, the reference execution time to which all strategies' execution times are compared is updated, signifying new fuzzy limits of the membership functions. Also, when a strategy takes longer than any other before to execute, the limit values that were assumed as representing a very big execution time are also updated. Regarding the modelling of the strategies' effectiveness, the same type of membership functions' update occurs, e.g. if strategies all start providing very bad results, comparing to what has been verified until that point, the values that were considered bad before, can now be considered a bit better, given the new circumstances. The detailed description of the $2 \mathrm{E}$ balance mechanism can be found in [47].

Besides the use of the $2 \mathrm{E}$ balance management mechanism, the execution time reduction issue has been dealt with by some other resources. Parallel computing has been used in the access to data. Although agents are, by their nature, parallel execution entities, there are still moments in which extra parallelism is necessary 
in order to decrease execution time. The access to huge amounts of data when necessary is one of the main times in which parallel computing is used.

A quick exhaustion of the resources of a machine can be led by the huge amount of parallel execution, and for this reason a mechanism that analyses the processing level of each machine available for the simulations, and distributes the agents accordingly, has been implemented. This is the mechanism used by the MIB Agent and Main Agent of MASCEM. The MIB Agent is a remote agent that reads the management information bases of each machine, generating a report that is after sent to the MASCEM's Main Agent. After receiving the report of each machine, the Main Agent redistributes MASCEM's agents by the available machines according to their characteristics and processing needs.

Another important contribution for the optimization of MASCEM's computational efficiency is the use of meta-heuristics, which diminishes the processing time of the required optimizations, Finally, the choice of the most adequate programming language for each of the developed algorithms has also been taken into account. MatLab has been used to implement mathematical calculations, like heuristics and optimization problems; Prolog for logic-based strategies; and C for other time-demanding modules. These developments also come to optimize the performance of the global Java based system, especially taking into account the need to support simulations involving several distinct agents from different simulators.

\subsection{Automatic Data Extraction}

Information is available online, concerning market proposals, including quantities and prices; accepted proposals and established market prices; proposals details; execution of physical bilateral contracts; statement outages, accumulated by unit type and technology; among others.

Since different operators make available different types of information, some of them providing even entities' technical characteristics and localization, it is important to be sure of the complete data available. This process implies analysing different types of files with different updating times that must be automatically downloaded, analysed and saved.

The automatic data extraction tool maintains a database updated with historical information from real electricity markets in order to be used by others systems, such as market simulators. For that, four major steps are considered: 
- Download data: the download of several files containing the new data;

- Parse data: extraction of the stored data from the downloaded files;

- Store data: storage of collected data in the database;

- Automatic data updates: define downloads periodicity automatically using machine learning techniques.

Since electricity markets information grows in a very dynamic way, the need for checking information updates is crucial. The implementation of a mechanism able to automatically check for information updates for downloading and performing database updates is of major importance, so that data can be used as soon as it is available online.

\section{Case Study}

The main goal of this case study is to test and demonstrate the enhanced simulation capabilities of MASCEM. Aiming at this goal, the performance of MASCEM is evaluated under two perspectives: (i) the quality of simulation results; (ii) the simulations' execution time.

\subsection{Definition}

In order to test the capability of MASCEM in representing the electricity market reality, the automatic data extraction tool, presented in [48], has been used to gather data from the Iberian electricity market - MIBEL [49], namely the players' characteristics (type of technology, installed capacity, number of groups, owner, etc.) and the market bids (energy, price and complex conditions for each negotiation period). The first undertaken simulation is referred to as Simulation 0 and uses the MIBEL players' data to create a set of MASCEM negotiating agents where each agent represents the characteristics and action behaviour of a real market player from MIBEL. This simulation allows testing the extent in which MASCEM is able to reflect the MIBEL electricity market reality when the number and characteristics of the involved agents are exactly equal to the players which participate in this market in the reality. The number of players that take part in this simulation is 1446 - the same players that have participated in the MIBEL day-ahead spot market in June, 1st 2012 [49]. The daily market has been chosen since it is the main MIBEL market (the market type with the largest amount of 
traded power) and also because it is easier to compare the results achieved by MASCEM simulation with the results of MIBEL for each day, in order to validate the MASCEM's market model. However, detailed results of the balancing market of MIBEL in MASCEM can be found in [13]. The integration of ancillary services can be seen in [50], using a case based on CAISO, where the transparency is greater, therefore being an efficient means to prove its validity. Concerning the ancillary services, there is no available data in MIBEL's web site, being impossible to compare the results and validate if the mechanism works correctly.

Taking into account the essential requirement that is the simulations' execution time, it becomes indispensable that MASCEM is able to represent realistic simulation scenarios which are able to summarize the reality, allowing undertaking valuable simulation studies in a reduced time frame, using a reduced amount of software agents. For this reason, two summarized simulation scenarios have been created, Using the complete set of agents that were created for Simulation 0, Scenario 1 bases the scenario summarization on the criterion of real players' dimension. This means that Scenario 1 contains only the agents that represent the real MIBEL players that negotiate the larger amounts of power in the electricity market. Three simulations are performed using Scenario 1:

- Simulation 1.1 includes the 48 players with the highest amount of trading power (24 sellers and 24 buyers);

- Simulation 1.2 includes the 24 players with the highest amount of trading power (12 sellers and 12 buyers);

- Simulation 1.3 includes the 12 players with the highest amount of trading power (6 sellers and 6 buyers).

With these simulations, based on Scenario 1, the performance of MASCEM in reflecting the reality is tested as the number of negotiating players decreases. This scenario also provides the means for testing whether the negotiating amount of power of real players is an adequate criterion for supporting the reduction in represented software agents, aiming at a critical reduction in simulations execution time.

Scenario 2 bases the reduction of the considered negotiating players in a different criterion: the bid price competitiveness. Under this scenario the selection of players to participate in the simulation enhances players' bid prices competitiveness, i.e. the selected players are the sellers that practice higher prices, and the buyers 
that are only willing to buy at the lower prices. Analogously to Scenario 1, also three simulations are performed using Scenario 2:

- $\quad$ Simulation 2.1 includes the 48 players with the most competitive prices ( 24 sellers and 24 buyers);

- $\quad$ Simulation 2.2 includes 24 players (12 sellers and 12 buyers);

- $\quad$ Simulation 2.3 includes the 12 players with the most competitive prices (6 sellers and 6 buyers).

The simulation scenarios refer to the 24 hourly periods of the MIBEL day-ahead spot market, of one negotiating day - June, 1st 2012. The market price results in each of the seven simulations (Simulation 0, three simulations using Scenario 1, and three simulations using Scenario 2) are compared to the real electricity market price that has been established during the same day, in order to compare the extent in which such summarized simulation scenarios are able to reflect the real electricity market outputs.

Finally, the execution time of the simulations is compared, in order to take conclusions on the advantage of using reduced, representative scenario, from a simulation efficiency standpoint.

All simulations have been performed on a computer with two Intel® Xeon ${ }^{\circledR}$ X5450 3.0GHz processors, each one with 2 cores, 4GB of random-access-memory and Windows Server 200832 bits operating system.

\subsection{Results - Established Market Prices}

Figure 3 presents the comparison between the market prices that have been achieved in each of the three simulations of Scenario 1, the real electricity market price of the considered day, and the market prices resulting from Simulation 0.

Comparison of the real electricity market prices from MIBEL day-ahead spot market in June 1 st 2012

with the market prices resulting from Simulation 0 and from the three simulations using Scenario 1

From Figure 3 it is visible that the market prices that resulted from Simulation 0 are exactly equal, in all 24 negotiating periods of the considered day, to those that resulted from the MIBEL day-ahead spot market. These results show that, by using the automatic data extraction tool, MASCEM is able to represent the real electricity market players as software agents, flawlessly. Also, when using the exact same number of correctly represented 
negotiating players, the simulated spot market results exactly as in the reality, proving the quality of negotiating models representation in MASCEM.

When reducing the number of represented players, the similarity of the results also decreases when compared to the real MIBEL outputs. When considering 48 simulated players (Simulation 1.1) the electricity market price curve follows the tendency of the real market price, accompanying the decrease in price in the first periods of the day, and the small peak in period 23. Reducing the number of players to 24 (Simulation 1.2) reflects into an increase of the price variation, distancing the resulting market price from the real MIBEL market price, although still following the real price tendency. Finally, when reducing the number of players to 12 (Simulation 1.3), it becomes obvious that the simulation is no longer capable of reflecting the reality. An enormous reduction of the number of players ( 6 buyers and 6 sellers, when the number of players participating in the MIBEL spot market is nearly 1500) leads to an incoherent representation of the market environment, when the selection of the players is performed depending on their negotiating amount of power.

Figure 4 presents the comparison between the market prices that have been achieved in each of the three simulations of Scenario 2 and the real electricity market price of the considered day.

Electricity market prices achieved in each of the three simulations undertaken under Scenario 2, and real electricity market price of MIBEL in June $1^{\text {st }}, 2012$

From Figure 4 it is visible that the market prices that resulted from each of the three simulations performed using Scenario 2 are, most of the times, equal to the real electricity market price of MIBEL for the same day. In fact, in the first two simulations, considering 48 and 24 players, respectively, only 3 from the 24 hourly periods of the day present market prices that do not coincide with the real prices. This results in a complete overlap of the real electricity market prices' curve in the graph of Figure 4. The third simulation, considering only 12 electricity market negotiating players, resulted in 8 hourly periods in which the achieved market price is different from the real one. However, even the referred 8 periods present very similar results when compared to real outputs of the MIBEL electricity market for the considered day - the difference is always inferior to 1 $€ / M W h$. 
These results show that MASCEM is able to properly represent the reality, even when the number of considered market negotiating agents is very small, as is the case of the third simulation - using only 12 agents. This representation is possible due to the simulator's capability in using real and adequate electricity market data, by using the automatic data extraction tool. The difference in results from the simulations of Scenario 1 to Scenario 2, even though using the same number of agents, show that having access to important data is not enough, but the proper use of these data is essential.

Table I shows a comparison of the error achieved in each of the seven simulations, when comparing the established electricity market price in each of the simulations, and the real market price of MIBEL during the 24 hours of the considered day. The comparison is performed using the Mean Absolute Percentage Error (MAPE).

From Table I it is visible that Simulation 0, reaching a market price equal to the real one during all the 24 periods of the considered day, achieved a MAPE value of 0 , showing the effectiveness of the used algorithm. Regarding the simulations performed using Scenario 1, the MAPE value clearly increases as the number of simulated players decreases. Finally, considering Scenario 2, the MAPE value also increases when the number of players present in the simulation decreases, however, this increase is very small, and the error values remain very low throughout all three simulations.

The difference in results from the simulations of Scenario 1 to those of Scenario 2 shows that the criteria for choosing the players involved in the simulations is critical to the outcomes and realism of the simulation environment. While in Scenario 1 the involved players negotiate huge amounts of power, their bids are highly conservative, in fact, most of the times the bigger buyers present their bids at the highest allowed price (180 $€ / \mathrm{MWh}$ ); while the bigger sellers present bids with the value of $0 € / \mathrm{MWh}$, in order to guarantee the transaction of the proposed amounts of power. This leads, however, to reaching very low market prices, or even null when considering only players that present such bids, as is the case of Simulation 1.3. On the other hand, in Scenario 2, the selection of the players depending on their bids competitiveness leads to achieving very close market prices to the reality. This occurs because the players involved in this simulation, even in Simulation 2.3, with 
only 12 simulated players, are the same ones that define the market price in the reality (the most competitive players - the ones with the bid prices closest to the market price).

\subsection{Results - Execution Time}

Table II shows the average execution time of MASCEM (in milliseconds) when performing simulations of the MIBEL day-ahead spot market with: 12 players, 24 players, 48 players, and 1446 players.

Average execution time of MASCEM (in milliseconds)

Table II

From Table II one can see that the difference in execution time from simulations with 12 players to 48 players is very small - about 1200 milliseconds. In fact, simulations in MASCEM with a rather reduced number of participating players are very fast. Executing a realistic electricity market simulation in 5 seconds is excellent. However, when increasing the number of considering players in order to represent all the real players means a huge increase in execution time - more than 1 minute for each simulation, which means that simulating a month takes more than half an hour. This becomes more than most people would be willing to wait for instantaneous results. This becomes even more evident when adding decision support capabilities to market players' behaviours. Note that the simulations presented in this case study include no decision support features for players' actions. Players' bids are exclusively based on the real MIBEL players auctioned prices and powers for the considered day, with no adaptation or learning features. Adding decision support capabilities for players’ actions leads to an enormous increase in execution time, as showed by Table III, for a reference simulation scenario of MASCEM with 24 negotiating players, with only one of these using ALBidS for decision support, for a single negotiation period.

Average execution time of MASCEM using ALBidS for decision support of players actions, with different percentages of preference the decision support effectiveness (in milliseconds)

Table III shows that using the decision support features of ALBidS at the maximum level of capabilities leads to a very significant increase of the execution time. The inclusion of all learning algorithms at their best 
performance is highly demanding. This type of decision support is critical when used by players for actual market negotiations. However, when MASCEM is used for studying purposes, where the extreme quality of the decision support is not critical, the use of the Efficiency/Effectiveness (2E) balance management mechanism allows using the decision support capabilities only in the required amount, in order to degrade the simulation execution time merely in the required measure. As seen by the case of $0 \%$ preference for the effectiveness of the decision support, where maximum preference is given to the computational efficiency of the simulation, the decision support is still used, but only in a very small extent, in a way that the increase in execution time is nearly not noticed. The advantages of using the $2 \mathrm{E}$ balance management mechanism are enormous, as it allows balancing the execution time requirements of each simulation, with the decision support needs.

Such drastic reductions in execution time are achieved by adapting each independent ALBidS' decision support strategy internally, forcing them to take less time to execute, when required, even if signifying a smaller effectiveness of results.

A good example of the execution time reduction when required is the execution of the ALBidS' Artificial Neural Network (ANN). The amount of training data is decreased when the demand for faster results increases, leading to a much faster training process, which still results in fairly good forecasts, achieved much more quickly than when aiming at the best possible forecast result. Table IV shows the comparison of the training process of the ANN when using different amounts of training data

ANN's average execution time, with and without parallel computing (in milliseconds)

The increase in execution time as the amount of training data increases is visible by Table IV. The use of a faster, yet less effective forecast, or a great forecast with the adjacent time demand, is decided by the $2 \mathrm{E}$ balance management mechanism. Another important aspect to be taken from Table IV is the huge decrease in execution time when using parallelism in the access to data. Although a multi-agent system, such as MASCEM and ALBidS, is by definition a parallel execution framework, where all agents are executed at the same time, there are still situations in which extra parallelism can be essential. The access to data, when it is done in a large scale, is a reference example, as can be seen by Table IV. 
Regarding the comparison with the previous version of MASCEM [4], the average execution time is now over ten times faster. Before the restructuring process that is presented in this paper, MASCEM agent framework was implemented on top of OAA (Open Agent Architecture), which led to several limitations, starting from the difficulties in making this platform a suitable simulation framework (requiring huge timedemanding computation just to adapt the OAA architecture to suit a simulation environment), and most importantly, limitations in the number of available players (MASCEM could not support the execution of more than 92 agents at simultaneously).

The average execution time of a simulation with 24 negotiating agents, with no decision support, in the previous version MASCEM is about 58364 milliseconds. This value is over ten times higher than an equivalent simulation in the new version of MASCEM (as presented in Table II). In fact, the execution time of such simulation (24 agents) in the previous version of MASCEM is close to the time amount required by a simulation with 1446 agents in the new version (Table II); and also close to the time demanded by a simulation in the new version using the decision support of ALBidS with $80 \%$ preference for effectiveness (Table III), which means using a very complete set of machine learning and decision support algorithms.

\section{Conclusions and Future Work}

This paper presented MASCEM, a multi-agent simulator of competitive electricity markets. MASCEM's restructuring resulted in an enhanced multi-agent simulator, which is able to interact and cooperate with other multi-agent societies through the use of ontologies that manage agents' communications. The integration of an automatic data extraction tool provides MASCEM the necessary information to recreate the electricity markets reality in a controlled environment. The purpose of each simulation is taken into account in order to adapt the balancing between the execution time and the quality of results, so that it most suits the expectation of the user. The possibility for the integration of such modules is accommodated by the architectural design of the simulator, which provides a flexible framework, which can be easily extended or integrated with other tools and platforms, increasing the scope of application of MASCEM, and with it, its usefulness for professionals and students of the field. 
The presented case study showed that the use of real data allows MASCEM to recreate real scenarios in a simulation environment. MASCEM's capability of representing the reality in a reduced and controlled way can be achieved even when using a summarized number of agents, as long as this summarization is done appropriately. The need for a coherent and automatic creation of scenarios is one of the most important future works to be taken into account. The automatic generation of scenarios must take into account, not only the dimension of the scenario (number of participating players), but also the most important characteristics for each simulation and that should be used as reference for the summarization of the real players in which the scenario is based.

The management of the simulations' execution time depending on the purpose of each simulation has also been demonstrated in the case study. The results have showed that MASCEM is able to reduce drastically its execution time when necessary, by reducing the execution time of some decision support strategies, or even, in the most extreme cases, excluding the most time-demanding decision support algorithms, so that the simulation can be executed as quickly as possible. In any case, the use of some implementation decisions, such as the parallelism in accessing data, the distribution of software agents by different available machines inside the network, and the implementation of algorithms in the most appropriate programming languages, make MASCEM's execution time be extremely fast by default. The difference of the average execution time of electricity market simulations in the novel version of MASCEM is very significant when compared to the previous version, as showed in the case study.

\section{Acknowledgements}

This work is supported by FEDER Funds through COMPETE program and by National Funds through FCT under the projects FCOMP-01-0124-FEDER: PEst-OE/EEI/UI0760/2015; PTDC/SEN-ENR/122174/2010; and PTDC/EEA-EEL/122988/2010.

\section{References}

[1] M. Shahidehpour, et al., "Market Operations in Electric Power Systems: Forecasting, Scheduling, and Risk Management”, Wiley-IEEE Press, pp. 233-274, 2002. 
[2] L. Meeus, K. Purchalaa, and R. Belmans, "Development of the Internal Electricity Market in Europe", The Electricity Journal, vol. 18, no. 6, pp. 25-35, 2005.

[3] I. Praça, C. Ramos, Z. Vale, and M. Cordeiro, "MASCEM: A Multi-Agent System that Simulates Competitive Electricity Markets", IEEE Intelligent Systems, vol. 18, No. 6, pp. 54-60, Special Issue on Agents and Markets, 2003.

[4] T. Pinto, et al., "A new approach for multi-agent coalition formation and management in the scope of electricity markets", Energy, vol. 36, no. 8, pp. 5004-5015, 2011.

[5] H. Li, and L. Tesfatsion, "Development of Open Source Software for Power Market Research: The AMES Test Bed", Journal of Energy Markets, vol. 2, no. 2, pp. 111-128, 2009.

[6] V. Koritarov,"Real-World Market Representation with Agents: Modeling the Electricity Market as a Complex Adaptive System with an Agent-Based Approach", IEEE Power\&Energy, pp.39-46, 2004.

[7] S. Cincotti, and G. Gallo, "Genoa Artificial Power-Exchange," Agents Artificial Intelligence, 2013.

[8] H. Morais, et al., "SOSPO-SP: Secure Operation of Sustainable Power Systems Simulation Platform for Real-Time System State Evaluation and Control", IEEE Transactions on Industrial Informatics, 2014.

[9] P. Vrba, et.al., "A Review of Agent and Service-oriented Concepts applied to Intelligent Energy Systems", IEEE Transactions on Industrial Informatics, 2014.

[10] H. Morais, et. al. "Multilevel Negotiation in Smart Grids for VPP Management of Distributed Resources", IEEE Intelligent Systems magazine, Special Issue "Sustainable Energy and Distributed AI”, vol. 27, no. 6, pág. 8-16, November - December, 2012.

[11]EMCC - European Market Coupling Company, homepage, http://www.marketcoupling.com/, accessed on March 2014.

[12] SGAM (Smart Grid Architectural Model), report, Available: http://ec.europa.eu/energy/gas_electricity/smartgrids/doc/xpert_group1_reference_architecture.pdf, accessed on May 2014.

[13] G. Santos, et.al., "Balancing Market Integration in MASCEM Electricity Market Simulator", IEEE Power and Energy Society General Meeting 2012, San Diego CA, USA, July 22 - 26, 2012.

[14] B. Canizes, et.al., "Mixed Integer Non Linear Programming and Artificial Neural Network Based Approach to Ancillary Services Dispatch in Competitive Electricity Markets", Applied Energy, vol. 108, pp. 261-270, August 2013.

[15]T. Sousa, et.al., "A Hybrid Simulated Annealing approach to handle Energy Resource Management considering an intensive use of Electric Vehicles", Energy, vol. 67, no. 1, pp. 81-96, April 2014.

[16] T. Pinto, et.al., "Short-term Wind Speed Forecasting using Support Vector Machines", IEEE SSCI 2014 (IEEE Symposium Series on Computational Intelligence), Orlando, Florida, USA.

[17] G. Santos, et.al., "MASCEM restructuring: Ontologies for scenarios generation in power systems simulators," Power and Energy Society General Meeting (PES), 2013 IEEE, vol., no., pp.1,5, 21-25 July 2013.

[18]D. Wenbin et al. "Automatically Generated Layered Ontological Models for Semantic Analysis of Component-Based Control Systems", IEEE Transactions on Industrial Informatics, v.9, i. 4, pp. 2124-2136, 2013.

[19]Z. Vale, T. Pinto, I. Praça, and H. Morais, "MASCEM: Electricity Markets Simulation with Strategic Agents", IEEE Intelligent Systems, vol. 26, no. 2, pág. 9-17, March-April 2011.

[20] P. Oliveira, T. Pinto, H. Morais, and Z. Vale. MASGriP: A multi-agent smart grid simulation platform. In Power and Energy Society General Meeting, 2012 IEEE, 2012.

[21]P. Leitão, et. al. "Past, Present, and Future of Industrial Agent Applications", IEEE Transactions on Industrial Informatics, vol.9, issue 4, pp. 2360-2372, 2013.

[22] J. Ferber, "Multi-Agent System: An Introduction to Distributed Artificial Intelligence", Harlow: Addison Wesley Longman, 1999.

[23] G. Santos, et.al., "Upper Ontology for Multi-Agent Energy Systems' Applications” in Distributed Computing and Artificial Intelligence - Advances in Intelligent Systems and Computing, Vol. 217, pp. 617-624, Springer Berlin Heidelberg, 2013.

[24] L. Gomes, et.al., "Distributed Intelligent Management of Microgrids using a Multi-Agent Simulation Platform", in Computational Intelligence Applications in Smart Grid (CIASG) of the IEEE SSCI 2014, Orlando, Florida, USA, 09-12 December, 2014.

[25] S. Harp, et al., "Sepia: A Simulator for Electric Power Industry Agents", IEEE Control Systems Magazine, vol. 20, no. 4, pp. $53-69,2000$. 
[26] R. Zimmermann, et al., "PowerWeb: a tool for evaluating economic and reliability impacts of electric power market designs", IEEE PES Power Systems Conference and Exposition, vol. 3, pp. 1562-1567, 2004.

[27] G. Migliavacca, "SREMS-electricity market simulator based Game Theory and incorporating network constraints", IEEE Power Tech, Lausanne, Swiss, 2007.

[28] G. Santos, et.al. "Complex Market integration in MASCEM electricity market simulator”,

EEM11 - 8th International Conference on European Energy Market, pp. 256-261, Zagreb, Croatia, 25-27, May, 2011.

[29] G. Santos, et.al. "Elspot: Nord Pool Spot Integration in MASCEM Electricity Market Simulator", MASGES 2014, on the Practical Applications of Agents and Multi-Agent Systems (PAAMS), 2014.

[30] G. Santos, et. al., "Multi-Agent Simulation of Continental, Regional, and Micro Electricity Markets", 23rd International Workshop on Database and Expert Systems Applications (DEXA 2012), Viena, Austria, 03-06 September, 2012.

[31] T. Pinto, et. al. "Strategic Bidding in Electricity Markets: An agent-based simulator with game theory for scenario analysis", Integrated Computer-Aided Engineering, IOS Press, vol. 20, no. 4, pp. 335-346, September 2013.

[32] Electricity Advisory Committee, Keeping the Lights On in a New World. January 2009. [Online]. Available: http://energy.gov/sites/prod/files/oeprod/DocumentsandMedia/adequacy_report_01-0909.pdf, accessed on May 2016.

[33] OMIE Report, Daily and Intraday Electricity Market Operating Rules, 2012. [Online]. Available: http://www.omel.es/files/reglas_agosto_2012_ingles.pdf, accessed on May 2016.

[34] OMIE, Markets and Products, Electricity Market, About our Market, available: http://www.omie.es/en/home/markets-and-products/about-our-market, accessed on May 2016.

[35] MIBEL Monthly Information, October 2015. [Online]. Available: http://www.mibel.com/index.php?mod=documentos\&mem=descargar\&fichero=documentos_BM_ MIBEL_201510_EN_f8ef347d.pdf, accessed on May 2016.

[36] E. Gamma, et.al., "Design Patterns: Elements of Reusable Object-Oriented Software", AddisonWesley Professional, November, 1994.

[37] E. Freeman, et.al. "Head First Design Patterns", O'Reilly Media, 2004.

[38] Foundation for Intelligent Physical Agents (FIPA). Agent management specification, 2002.

[39] Foundation for Intelligent Physical Agents (FIPA). FIPA ACL message structure specification, 2002.

[40] Java Agent Development Framework (JADE): Available: http://jade.tilab.com/.

[41] G. Santos, et al, Multi-Agent Simulation of Competitive Electricity Markets: Autonomous systems cooperation for European Market modeling, Energy Conversion and Management, vol. 99, pp. 387 399, July 2015.

[42] T. Pinto, et al., Smart Grid and Electricity Market joint Simulation using complementary MultiAgent platforms, 2015 IEEE PowerTech Eindhoven, Eindhoven, Netherlands, 29 June - 2 July, 2015.

[43] G. Santos, et al., Agent-based Smart Grid Market Simulation with connection to real infrastructures Highlights of Practical Applications of Agents, Multi-Agent Systems, and Sustainability - The PAAMS Collection Communications in Computer and Information Science, vol. 524, pp. 283-295, Springer International Publishing, 2015.

[44]Foundation for Intelligent Physical Agents (FIPA), FIPA Ontology Service. [Online]. Available: http://www.fipa.org/.

[45] V. M. Catterson, P. C. Baker, E. M. Davidson, S. D. J. McArthur, "An upper ontology for power engineering applications", available from http://sites.ieee.org/pes-mas/April 2010.

[46] G. Rigatos, "Adaptive fuzzy control for differentially flat MIMO nonlinear dynamical systems", Integrated Computer-Aided Engineering, IOS Press, vol. 20, no. 2, pp. 111-126, 2013.

[47] T. Pinto. et al., "Bid Definition Method for Electricity Markets Based on an Adaptive Multiagent System", Advances on Practical Applications of Agents and Multiagent Systems, Springer Berlin / Heidelberg, vol. 88, pp. 309-316, 2011.

[48] I. F. Pereira, et.al., "Data Extraction Tool to Analyse, Transform and Store Real Data from Electricity Markets" in Advances in Intelligent Systems and Computing, S. Omatu, et al. (Eds), pp. 387-395, Springer International Publishing, 2014. 
[49] MIBEL - Iberian Electricity Market Operator, homepage, http://www.omie.es, accessed on April 2014.

[50] Soares T., Santos G., Faria P., Pinto T., Vale Z. and Morais H. Integration in MASCEM of the Joint Dispatch of Energy and Reserves Provided by Generation and Demand Resources. ISAP 2013 - 17th International Conference on Intelligent System Applications to Power Systems, Tokyo, Japan, 01-04 July, 2013.

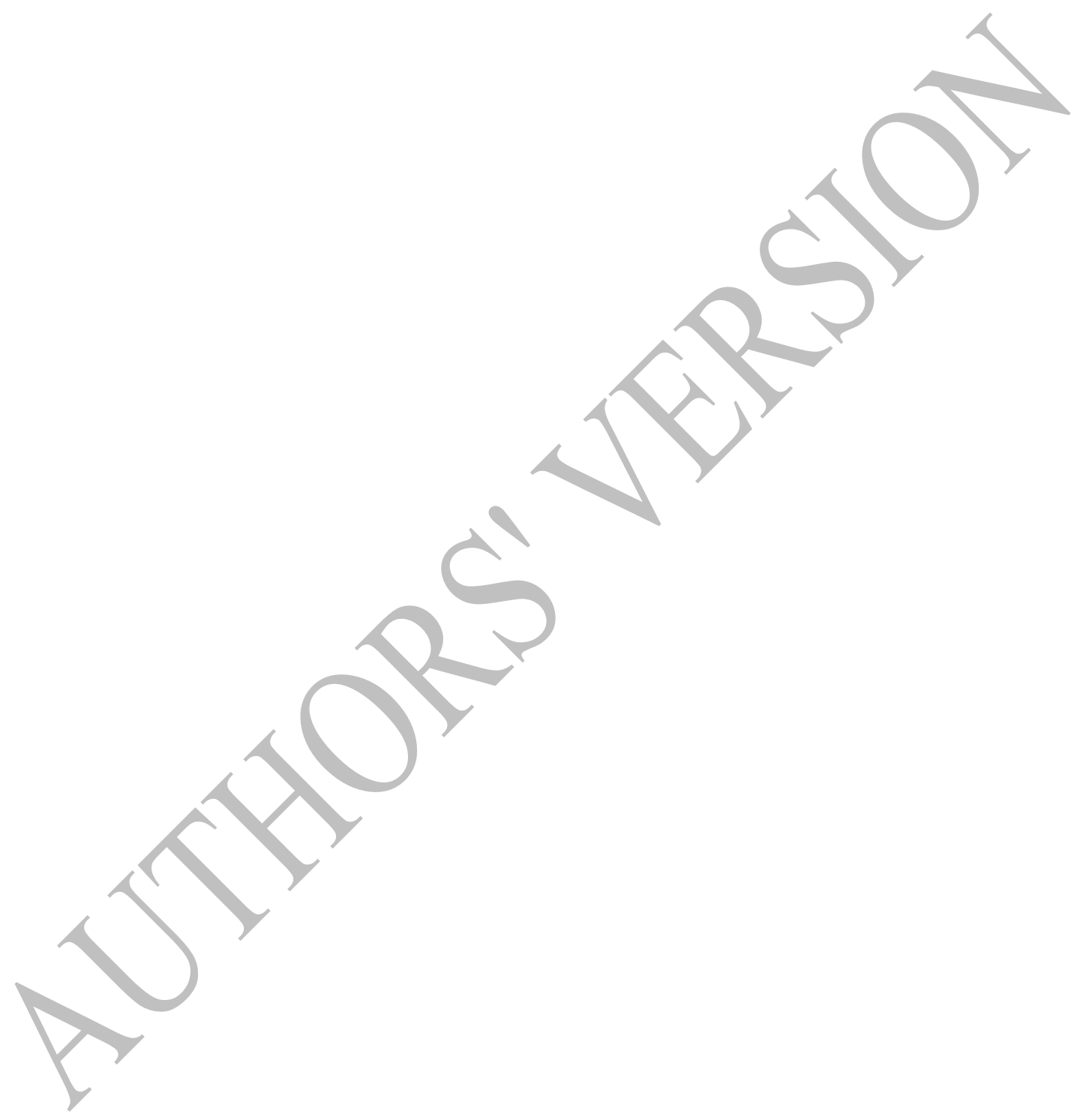




\section{List of Figures}
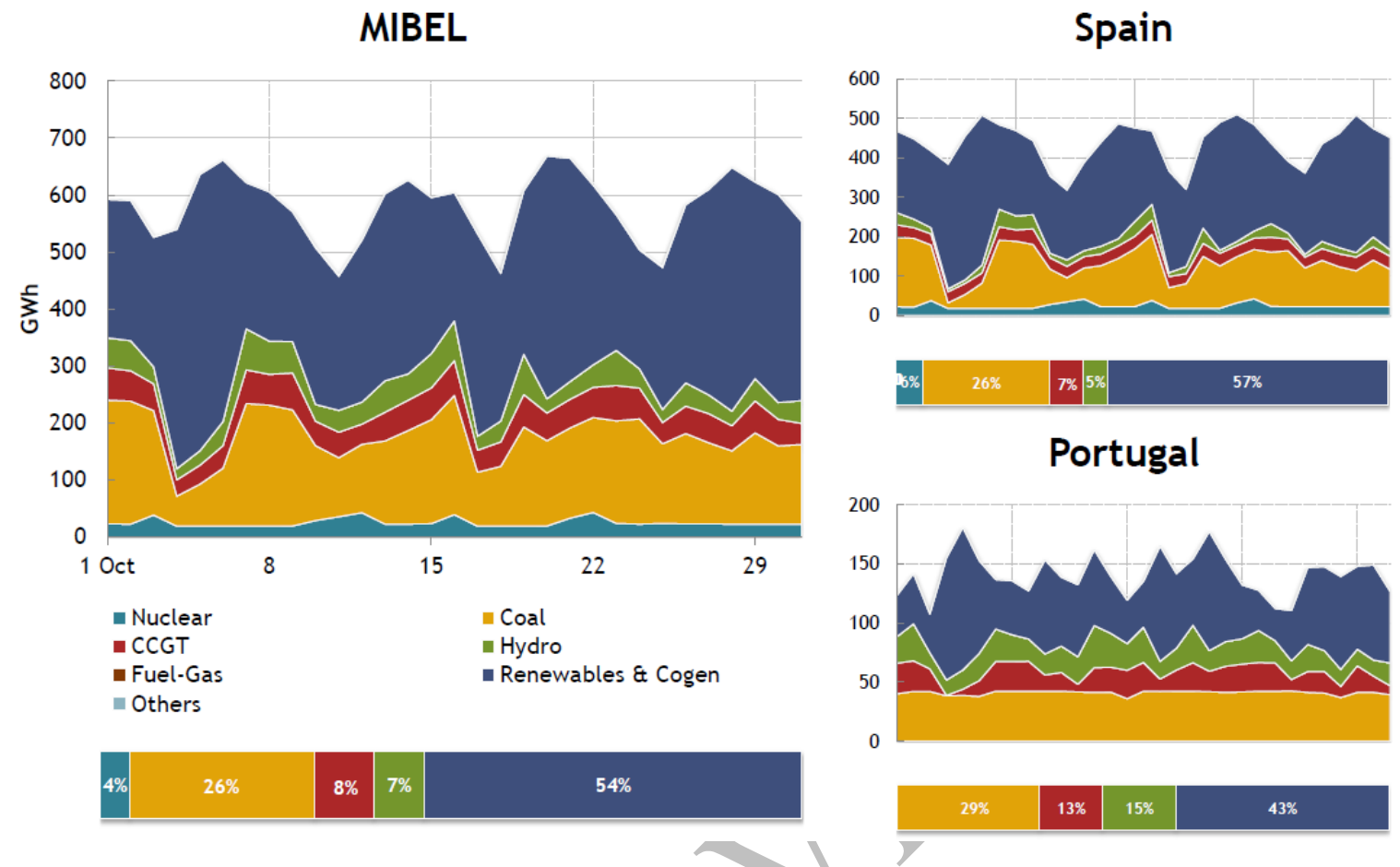

Fig. 1. Data on the energy placed in the day-ahead market, October 2016 [35]

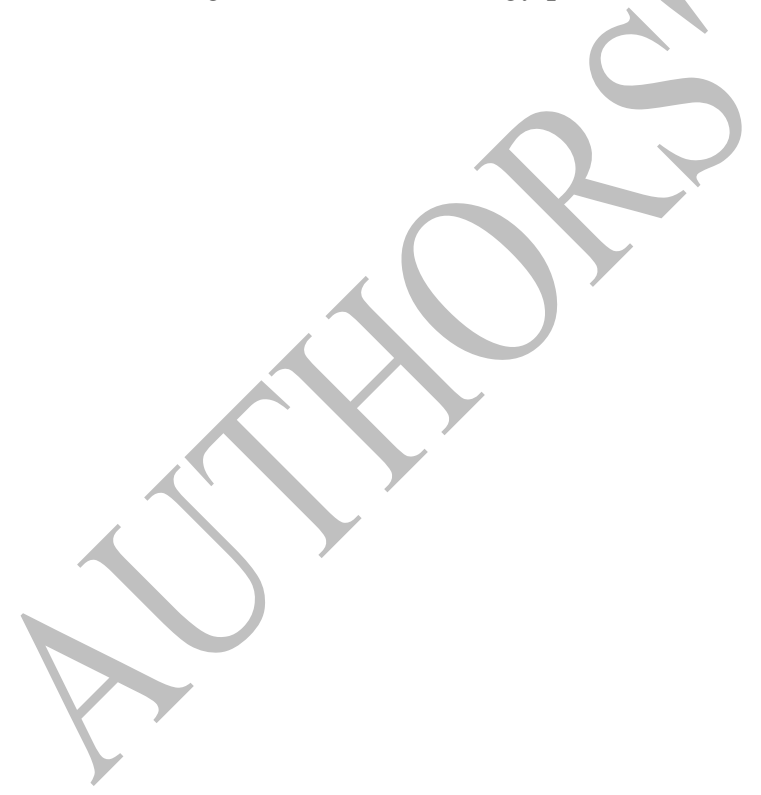




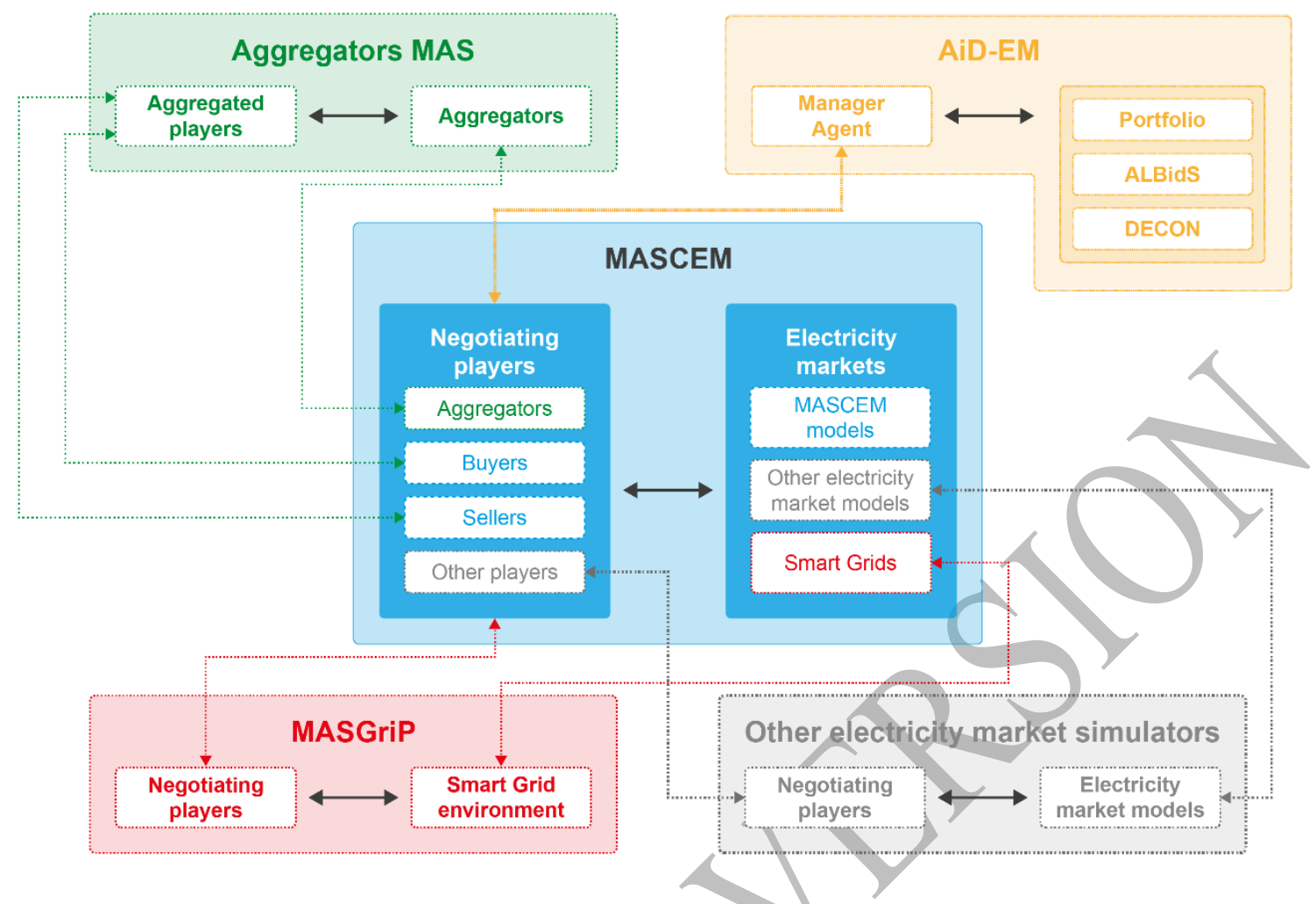

Fig. 2. Collaboration between independent multi-agent systems

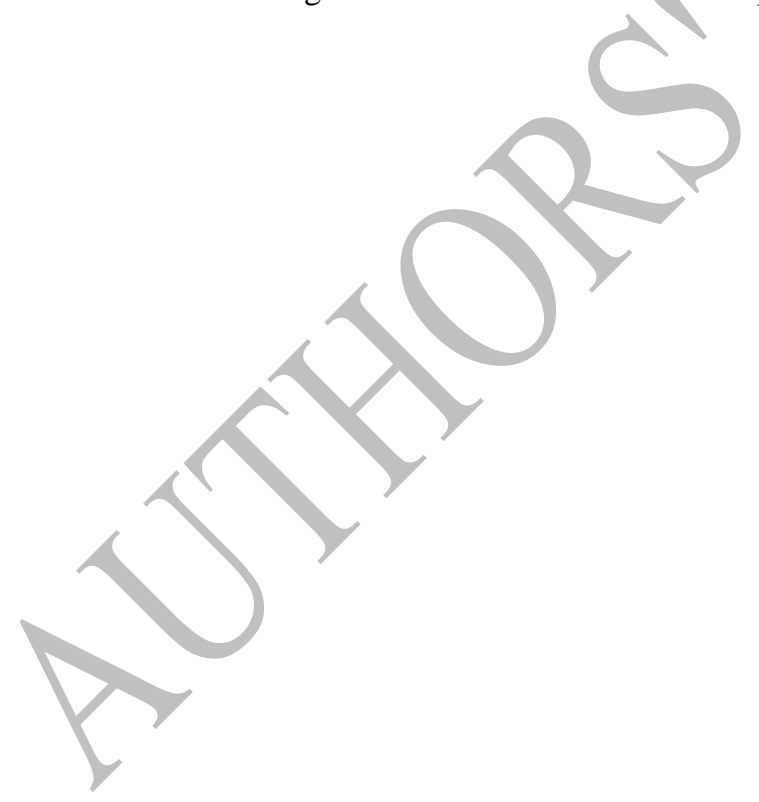



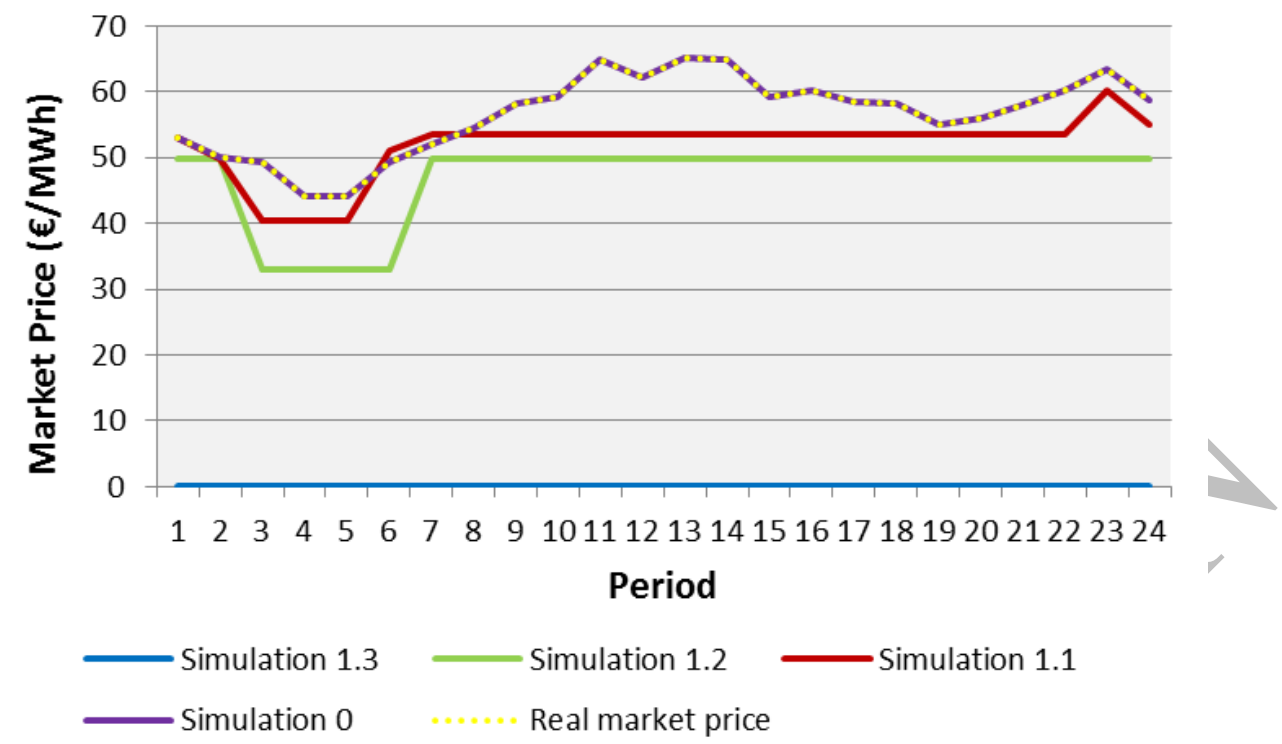

Fig. 3. Comparison of the real electricity market prices from MIBEL day-ahead spot market in June $1^{\text {st }} 2012$ with the market prices resulting from Simulation 0 and from the three simulations using Scenario 1 


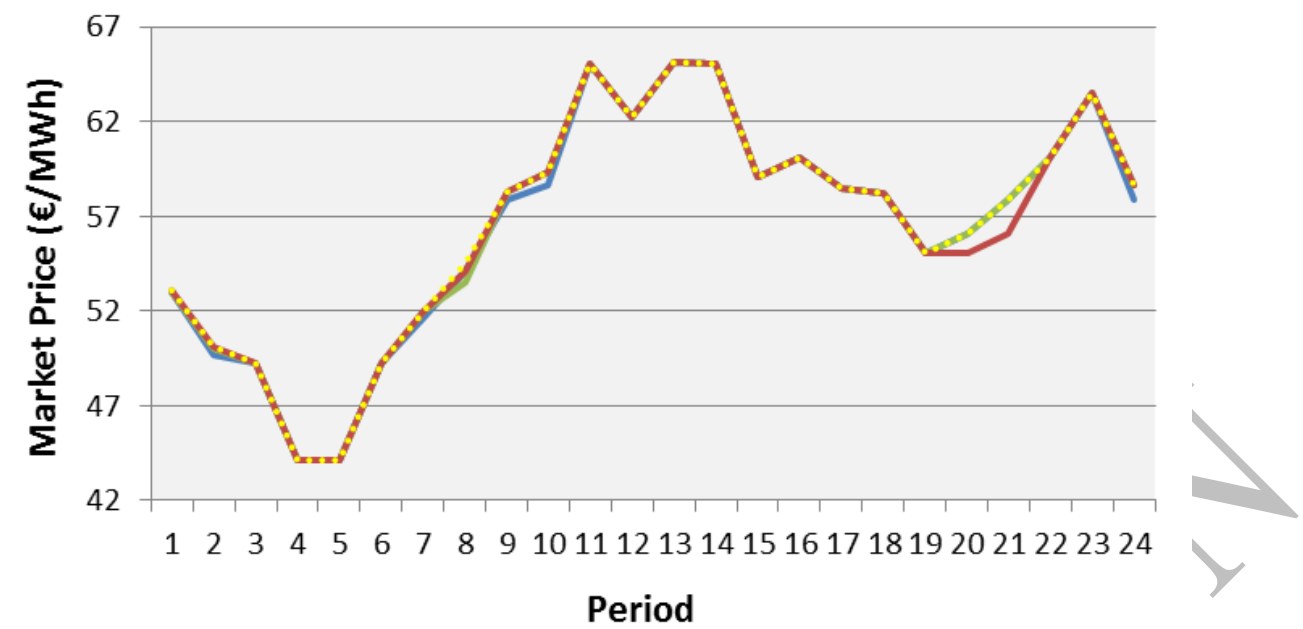

- Simulation 2.3 (12 players) - Simulation 2.2 (24 players)
- Simulation 2.1 (48 players) $\cdots \cdots$ Real market price

Fig. 4. Electricity market prices achieved in each of the three simulations undertaken under Scenario 2, and real electricity market price of MIBEL in June $1^{\text {st }}, 2012$ 
List of Tables

TABLE I - SIMULATED MARKET PRICE MAPE VALUES (\%)

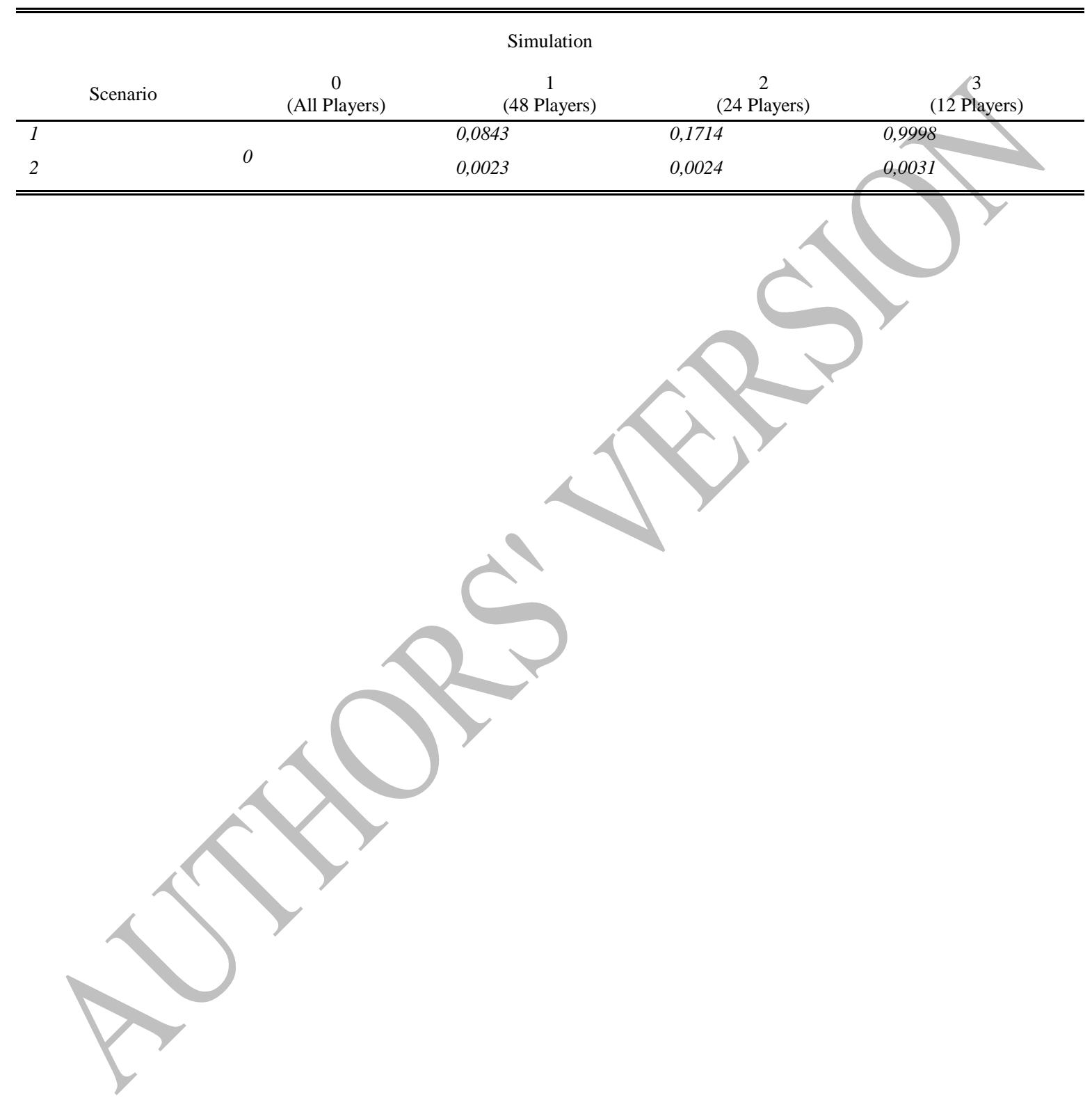


TABLE II - AVERAGE EXECUTION TIME OF MASCEM (IN MILLISECONDS)

\begin{tabular}{lcccc}
\hline \hline & 1446 Players & 48 Players & 24 Players & 12 Players \\
\hline 65327 & 5628 & 4841 & 4426 \\
\hline \hline
\end{tabular}

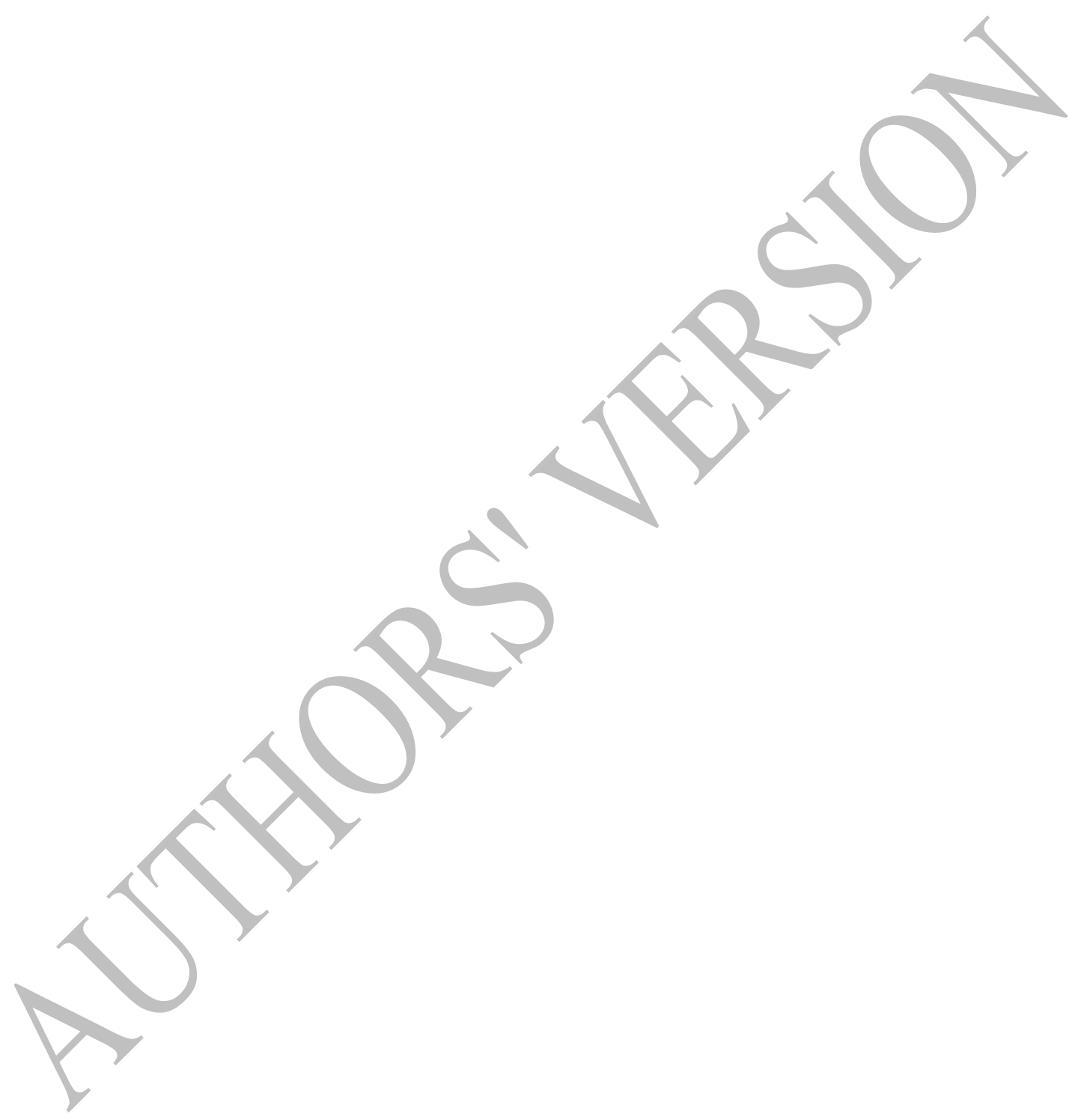


TABLE III - AVERAGE EXECUTION TIME OF MASCEM USING ALBIDS FOR DECISION SUPPORT OF PLAYERS ACTIONS, WITH DIFFERENT PERCENTAGES OF PREFERENCE THE DECISION SUPPORT EFFECTIVENESS (IN MILLISECONDS)

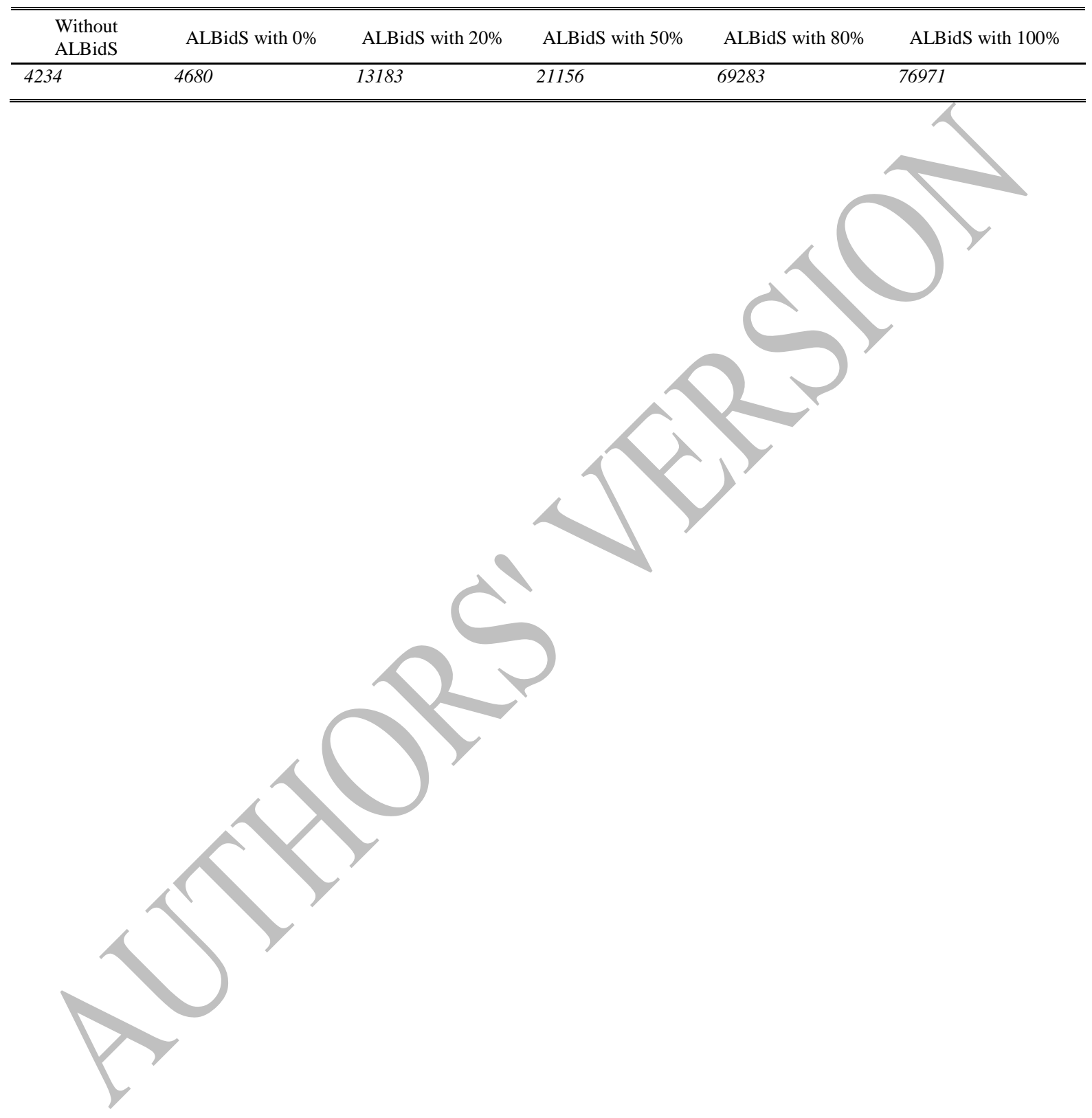


TABLE IV - ANN'S AVERAGE EXECUTION TIME, WITH AND WITHOUT PARALLEL COMPUTING (IN MILLISECONDS)

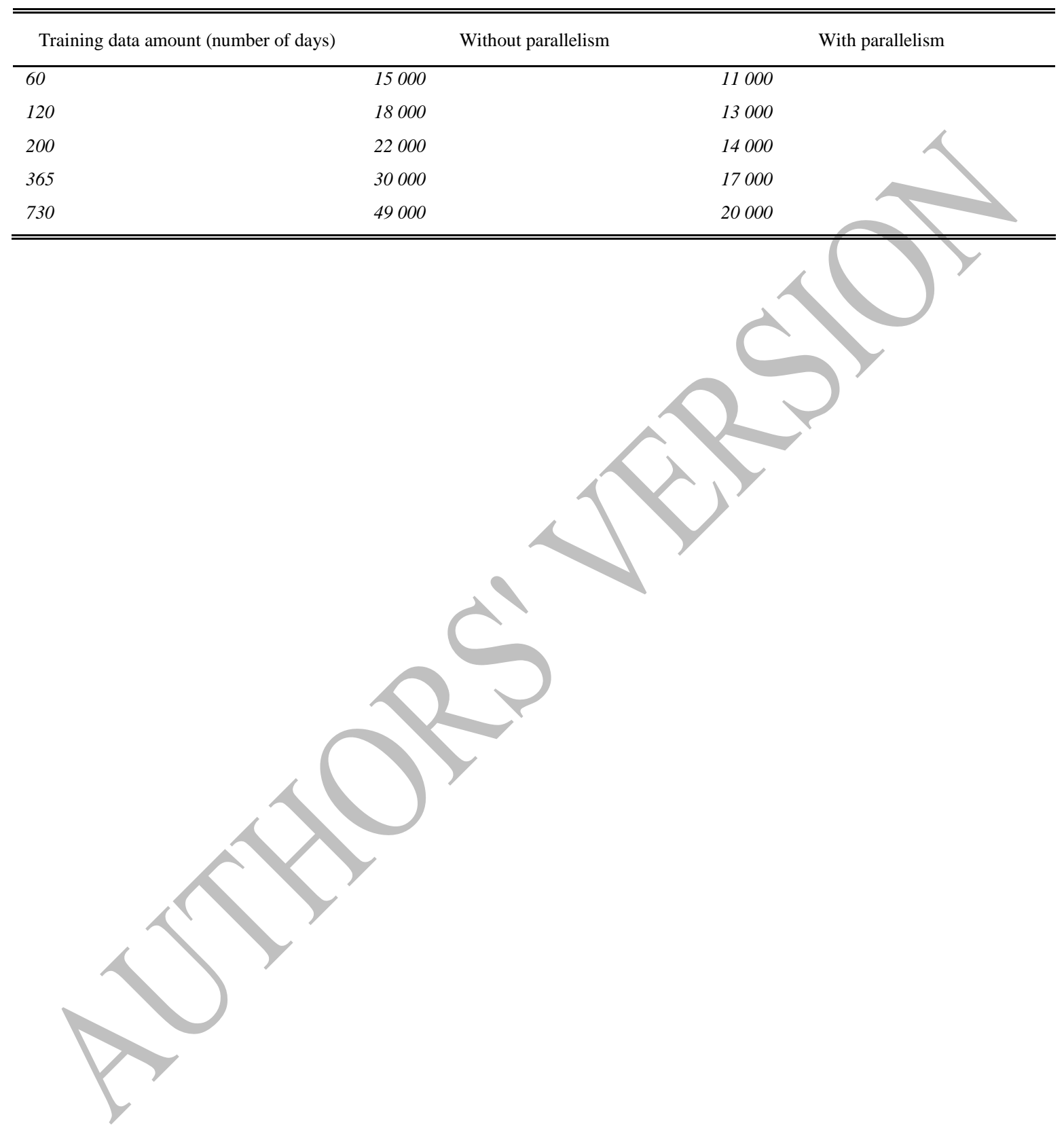

